\title{
AN EXTENSION OF THE THEOREM ON PRIMITIVE DIVISORS IN ALGEBRAIC NUMBER FIELDS
}

\author{
A. SCHINZEL
}

In memory of D. H. Lehmer

\begin{abstract}
The theorem about primitive divisors in algebraic number fields is generalized in the following manner. Let $A, B$ be algebraic integers, $(A, B)=$ $1, A B \neq 0, A / B$ not a root of unity, and $\zeta_{k}$ a primitive root of unity of order $k$. For all sufficiently large $n$, the number $A^{n}-\zeta_{k} B^{n}$ has a prime ideal factor that does not divide $A^{m}-\zeta_{k}^{j} B^{m}$ for arbitrary $m<n$ and $j<k$.
\end{abstract}

The analogue of Zsigmondy's theorem in algebraic number fields [3] asserts the following.

If $A, B$ are algebraic integers, $(A, B)=1, A B \neq 0$, and $A / B$ of degree $d$ is not a root of unity, there exists a constant $n_{0}(d)$ such that for $n>n_{0}(d)$, $A^{n}-B^{n}$ has a prime ideal factor that does not divide $A^{m}-B^{m}$ for $m<n$.

This theorem will be extended as follows:

Theorem. Let $K$ be an algebraic number field, $A, B$ integers of $K,(A, B)=$ $1, A B \neq 0, A / B$ of degree $d$ not a root of unity, and $\zeta_{k}$ a primitive $k$ th root of unity in $K$. For every $\varepsilon>0$ there exists a constant $c(d, \varepsilon)$ such that if $n>c(d, \varepsilon)(1+\log k)^{1+\varepsilon}$, there exists a prime ideal of $K$ that divides $A^{n}-\zeta_{k} B^{n}$, but does not divide $A^{m}-\zeta_{k}^{j} B^{m}$ for $m<n$ and arbitrary $j$.

The above theorem implies the finiteness of the number of solutions of generalized cyclotomic equations considered by Browkin [1, p. 236].

The proof will follow closely the proof given in [3]. Let $\mathbb{Q}(A / B)=K_{0}$, $\frac{A}{B}=\frac{\alpha}{\beta}$, where $\alpha, \beta \in K_{0}, \alpha, \beta$ are integers, and $(\alpha, \beta)=\mathfrak{d}$. Let $S$ and $S_{0}$ be the set of all isomorphic injections of $K_{0}\left(\zeta_{k}\right)$ and $K_{0}$, respectively, in the complex field, and set

$$
w(\alpha / \beta)=\log \prod_{\sigma \in S_{0}} \max \left\{\left|\alpha^{\sigma}\right|,\left|\beta^{\sigma}\right|\right\}-\log N \mathfrak{d},
$$

where $N$ denotes the absolute norm in $K_{0}$. Here, $w(\alpha / \beta)$ is the logarithm of the Mahler measure of $\alpha / \beta$ and so it is independent of the choice of $\alpha, \beta$ in $K_{0}$.

Lemma 1. If $|\alpha|=|\beta|$, but $\alpha / \beta$ is not a root of unity, then

$$
\log \left|\alpha^{n}-\zeta_{k} \beta^{n}\right|=n \log |\beta|+O(d+w(\alpha / \beta)) \log k n,
$$

Received by the editor July 27, 1992.

1991 Mathematics Subject Classification. Primary 11R04. 
where the constant in the O-symbol depends only on $d$ and is effectively computable.

Lemma 2. If $|\alpha| \neq|\beta|$, then

$$
\log \left|\alpha^{n}-\zeta_{k} \beta^{n}\right|=n \log \max \{|\alpha|,|\beta|\}+O\left(d^{2}+d w(\alpha / \beta)\right),
$$

where the constant in the O-symbol is absolute and effectively computable.

The next lemma is just quoted from [3], where it occurs as Lemma 4.

Lemma 3. Let $\phi_{n}(x, y)$ be the nth cyclotomic polynomial in homogeneous form. If $\mathfrak{P}$ is a prime ideal of $K, n>2\left(2^{d}-1\right), \mathfrak{P} \mid \phi_{n}(A, B)$, and $\mathfrak{P}$ is not a primitive divisor of $A^{n}-B^{n}$, then

$$
\operatorname{ord}_{\mathfrak{P}} \phi_{n}(A, B) \leq \operatorname{ord}_{\mathfrak{P}} n \text {. }
$$

Finally, we prove

Lemma 4. Let

$$
\psi_{n}\left(x, y ; \zeta_{k}\right)=\prod_{\substack{(j, n)=1 \\ j \equiv 1 \bmod k}}\left(x-\zeta_{k n}^{j} y\right)
$$

We have

$$
\psi_{n}\left(x, y ; \zeta_{k}\right)=\prod_{\substack{m \mid n \\(m, k)=1}}\left(x^{n / m}-\zeta_{k}^{\bar{m}} y^{n / m}\right)^{\mu(m)},
$$

where $m \bar{m} \equiv 1 \bmod k$ and

$$
\operatorname{deg} \psi_{n}=\varphi(n) \frac{(k, n)}{\varphi((k, n))}
$$

Proof. The right-hand side of (1) can be written as

$$
\prod_{\substack{m \mid n \\(m, k)=1}} \prod_{i=0}^{n / m-1}\left(x-\zeta_{n / m}^{i} \zeta_{k n / m}^{\bar{m}} y\right)^{\mu(m)}
$$

A factor $x-\zeta_{k n}^{j} y$ occurs in this product with the exponent

$$
E=\sum_{\substack{m \mid n \\(m, k)=1}} \mu(m) \sum_{\substack{i=0 \\ m(k i+\bar{m}) \equiv j \bmod k n}}^{n / m-1} 1 .
$$

Now,

$$
\sum_{\substack{i=0 \\ m(k i+\bar{m}) \equiv j \bmod k n}}^{n / m-1} 1= \begin{cases}\sum_{\substack{i=0 \\ k i+\bar{m} \equiv j / m \bmod k n / m}}^{n / m-1} 1 \text { if } m \mid j, \\ 0 & \text { otherwise }\end{cases}
$$

and if $m \mid j$,

$$
\sum_{\substack{i=0 \\ k i+\bar{m} \equiv j / m \bmod k n / m}}^{n / m-1} 1= \begin{cases}1 & \text { if } j \equiv 1 \bmod k \\ 0 & \text { otherwise }\end{cases}
$$


Hence,

and finally

$$
E= \begin{cases}\sum_{m|n, m| j} \mu(m) & \text { if } j \equiv 1 \bmod k \\ 0 & \text { otherwise }\end{cases}
$$

$$
E= \begin{cases}1 & \text { if }(n, j)=1, j \equiv 1 \bmod k, \\ 0 & \text { otherwise },\end{cases}
$$

which proves the first part of the lemma.

In order to prove the second part, we notice that there are exactly $\varphi(n) \frac{(k, n)}{\varphi((k, n))}$ positive integers $j \leq k n$ such that $(n, j)=1, j \equiv 1 \bmod k$.

Lemma 5. For every $\varepsilon>0$ there exists $c(d, \varepsilon)$ such that, if

$$
n>c(d, \varepsilon)(1+\log k)^{1+\varepsilon},
$$

then we have

$$
\left|N_{K / \mathbb{Q}} \psi_{n}\left(A, B ; \zeta_{k}\right)\right|>(n k)^{[K: \mathbb{Q}]}
$$

Proof. By Lemma 4,

$$
\psi_{n}\left(A, B ; \zeta_{k}\right)=\left(\frac{B}{\beta}\right)^{\phi(n)(k, n) / \phi((k, n))} \psi_{n}\left(\alpha, \beta ; \zeta_{k}\right),
$$

and since $\left(\frac{B}{\beta}\right)=\mathfrak{d}^{-1}$, we have

$$
\begin{aligned}
& \left(\psi_{n}\left(A, B ; \zeta_{k}\right)\right)=\mathfrak{d}^{-\varphi(n)(k, n) / \varphi((k, n))} \psi_{n}\left(\alpha, \beta ; \zeta_{k}\right), \\
& \frac{1}{\left[K: K_{0}\left(\zeta_{k}\right)\right]} \log \left|N_{K / \mathbb{Q}} \psi_{n}\left(A, B ; \zeta_{k}\right)\right| \\
& =\log \left|N_{K_{0}\left(\zeta_{k}\right) / \mathbb{Q}} \psi_{n}\left(\alpha, \beta ; \zeta_{k}\right)\right|-\left[K_{0}\left(\zeta_{k}\right): K_{0}\right] \varphi(n) \frac{(k, n)}{\varphi((k, n))} \log N \mathfrak{d} \\
& =\sum_{\sigma \in S} \sum_{\substack{m \mid n \\
(m, k)=1}} \mu(m) \log \left|\left(\alpha^{\sigma}\right)^{n / m}-\zeta_{k}^{\bar{m}}\left(\beta^{\sigma}\right)^{n / m}\right| \\
& -\left[K_{0}\left(\zeta_{k}\right): K_{0}\right] \varphi(n) \frac{(k, n)}{\varphi((k, n))} \log N \mathfrak{d} \\
& =\sum_{\sigma \in S} \sum_{\substack{m \mid n \\
(m, k)=1}} \mu(m)\left(\frac{n}{m} \log \max \left\{\left|\alpha^{\sigma}\right|,\left|\beta^{\sigma}\right|\right\}+O\left(d+w\left(\frac{\alpha}{\beta}\right)\right) \log k n\right) \\
& -\left[K_{0}\left(\zeta_{k}\right): K_{0}\right] \varphi(n) \frac{(k, n)}{\varphi((k, n))} \log N \mathfrak{d} \\
& =\left[K_{0}\left(\zeta_{k}\right): K_{0}\right]\left(\varphi(n) \frac{(k, n)}{\varphi((k, n))} w\left(\frac{\alpha}{\beta}\right)+O\left(d+w\left(\frac{\alpha}{\beta}\right)\right) 2^{\nu(n)} \log k n\right),
\end{aligned}
$$

where the constant in $O$ depends only on $d$ and is effectively computable. Now, by Dobrowolski's theorem [2], if $\alpha / \beta$ is an integer, then

$$
\begin{aligned}
w\left(\frac{\alpha}{\beta}\right) & =\log \prod_{\sigma \in S_{0}} \max \left\{\left|\frac{\alpha^{\sigma}}{\beta^{\sigma}}\right|, 1\right\} \\
& \geq \log \left(1+c_{1}\left(\frac{\log \log e d}{\log d}\right)^{3}\right) \geq c_{2}\left(\frac{\log \log e d}{\log d}\right)^{3},
\end{aligned}
$$

where $c_{1}$ and $c_{2}$ are absolute constants. 
If $\alpha / \beta$ is not an integer, then $(\beta) \neq \mathfrak{d}$ and

$$
w\left(\frac{\alpha}{\beta}\right) \geq \log N \beta-\log N \mathfrak{d} \geq \log 2 .
$$

Thus, in both cases,

$$
w\left(\frac{\alpha}{\beta}\right) \geq c_{2}\left(\frac{\log \log e d}{\log d}\right)^{3}
$$

provided $c_{2} \leq \log 2$.

Since for every $\varepsilon>0$

$$
\frac{\varphi(n)}{2^{\nu(n)}}>c_{3}(\varepsilon) n^{1-\varepsilon}
$$

it follows that for $n>c(d, \varepsilon)(1+\log k)^{1+\varepsilon}$

$$
\log \left|N_{K / \mathbb{Q}} \psi_{n}\left(A, B ; \zeta_{k}\right)\right|>[K: \mathbb{Q}] \log n k,
$$

which proves the lemma.

Proof of the theorem. By Lemma 5, for $n>c(d, \varepsilon)(\log k)^{1+\varepsilon}$ we have (2), and thus $\psi_{n}\left(A, B ; \zeta_{k}\right)$ has a prime ideal factor $\mathfrak{P}$ in $K$ such that

$$
\operatorname{ord}_{\mathfrak{P}} \psi_{n}\left(A, B ; \zeta_{k}\right)>\operatorname{ord}_{\mathfrak{P}} k n \text {. }
$$

But $\mathfrak{P}\left|\psi_{n}\left(A, B ; \zeta_{k}\right)\right| \phi_{k n}(A, B)$, hence by Lemma 3 we have that $\mathfrak{P}$ is a primitive prime divisor of $A^{k n}-B^{k n}$ and thus does not divide $A^{m}-\zeta_{k}^{j} B^{m}$ for $m<n$ and arbitrary $j$. On the other hand,

$$
\mathfrak{P}\left|\psi_{n}\left(A, B ; \zeta_{k}\right)\right| A^{n}-\zeta_{k} B^{n},
$$

thus $\mathfrak{P}$ has the desired property.

\section{BIBLIOGRAPHY}

1. J. Browkin, K-theory, cyclotomic equations, and Clausen's function, Chapter 11, Math. Surveys Monographs (L. Lewin, ed.), vol. 37, Amer. Math. Soc., Providence, RI, 1991, pp. 233-273.

2. E. Dobrowolski, On a question of Lehmer and the number of irreducible factors of a polynomial, Acta Arith. 34 (1979), 391-401.

3. A. Schinzel, Primitive divisors of the expression $A^{n}-B^{n}$ in algebraic number fields, J. Reine Angew. Math. 268/269 (1974), 27-33.

Institute of Mathematics, Polish Academy of Sciences, P.O. Box 137, 00-950 Warsaw, Poland

E-mail address: schinzel@impan.impan.gov.pl 